$\mathrm{e}^{+} \mathrm{e}^{-}$Collisions from Phi to Psi 2013 (PHIPSI2013)

International Journal of Modern Physics: Conference Series

Vol. 35 (2014) 1460449 (6 pages)

(C) The Author

DOI: $10.1142 /$ S2010194514604499

\title{
STATUS OF SuperKEKB AND BELLE II
}

\author{
B.A. SHWARTZ \\ on behalf of the Belle II collaboration \\ Budker Institute of Nuclear Physics of SB RAS, Novosibirsk, 630090, Russia \\ Novosibirsk State University, Novosibirsk, 630090, Russia \\ shwartz@inp.nsk.su
}

Published 18 December 2014

The current status of the SuperKEKB/Belle II project which is under construction now at KEK is reported. Main motivations and goals of this project are discussed as well.

\section{Introduction}

During a decade from 2000 to 2010 large number of results on particle physics were produced by two experiments, Belle ${ }^{1}$ and BaBar, ${ }^{2}$ operated at two energyasymmetric $e^{+} e^{-}$B-factories-KEKB ${ }^{3}$ and PEP-II. ${ }^{4}$ The world highest collider luminosity, $2.1 \times 10^{34} \mathrm{~cm}^{-2} \mathrm{~s}^{-1}$, was achieved by KEKB. Integrated luminosity collected by both detectors exceeds $1500 \mathrm{fb}^{-1}$.

Motivated by the success of the KEKB/Belle experiment, the new advanced project, KEKB II/Belle II, was accepted. The KEKB II luminosity will exceed the previous one by about 40 times. In many aspects, Belle II will have considerably better performance than Belle.

Physics motivations as well as main features of the collider and detector are discussed in this report.

\section{Physics Motivations}

The primary goal of the Belle and BaBar experiments was to discover the $\mathrm{CP}$ violation in $B$ mesons and to measure the parameters of $\mathrm{CPV}$. This was achieved by both experiments in 2001 when time dependent CP asymmetry was observed in the decay $B^{0} \rightarrow J / \Psi K^{0}{ }^{5}$ The value of this asymmetry is determined by the angle $\phi_{1}$ of the unitarity triangle of the CKM matrix. At present the most precise measurement of the $\sin 2 \phi_{1}$ made by Belle is: $\sin 2 \phi_{1}=0.667 \pm 0.023$ (stat) \pm 0.012 (syst). ${ }^{6}$ By now

This is an Open Access article published by World Scientific Publishing Company. It is distributed under the terms of the Creative Commons Attribution 3.0 (CC-BY) License. Further distribution of this work is permitted, provided the original work is properly cited. 
$\mathrm{CP}$ violation including direct $\mathrm{CP}$ violation was observed in many other $B$ mesons decay modes.

In general, in these experiments Standard Model (SM) was confirmed with the Kobayashi-Maskawa mechanism of CP violation. However, present uncertainties in the CKM matrix elements still leave certain room for New Physics (NP). At $50 \mathrm{ab}^{-1}$, which should be reached with SuperKEKB, uncertainties in the $\phi_{2}$ and $\phi_{3}$ angles will be improved to about $1.5^{\circ}$. The $\bar{\rho}$ and $\bar{\eta}$ parameters of the unitarity triangle will be determined with 6-8 times better accuracy than now.

A search for NP, i.e. phenomena which are not described by the SM, becomes the most important task for the Belle II experiment.

Evidences of the NP will be searched not only in the B mesons decays but, also in the charm sector and $\tau$ lepton decays. Other studies like precise measurement of the hadronic cross sections in $\gamma \gamma$ and $e^{+} e^{-}\left(\gamma_{I S R}\right)$ processes are important issues of the Belle II program. So wide researches area becomes possible because of clean event environment and well defined initial state in the $e^{+} e^{-}$experiments as well as high luminosity and general purpose detector.

\section{SuperKEKB Collider}

The new project is based on the so called "Nano-Beam" scheme, first proposed in. ${ }^{8}$

Main parameters of the new collider are listed in Table 1

Table 1. Comparison of the main parameters of SuperKEKB and previous KEKB colliders

\begin{tabular}{lcc}
\hline & KEKB (achieved) & SuperKEKB \\
\hline Energy, $\mathrm{GeV}\left(e^{+} / e^{-}\right)$ & $3.5 / 8.0$ & $4.0 / 7.0$ \\
Beam current, A & $1.64 / 1.19$ & $3.6 / 2.6$ \\
Vertical $\beta$ function at IP, mm & $5.9 / 5.9$ & $0.27 / 0.30$ \\
Beam-beam parameter, $\xi_{y}$ & $0.129 / 0.090$ & $0.09 / 0.081$ \\
Beam sizes $\left(\sigma_{x}, / \sigma_{y}\right), \mu \mathrm{m}$ & $100 / 2$ & $10 / 0.06$ \\
Luminosity, $10^{34} \mathrm{~cm}^{-2} \mathrm{~s}^{-1}$ & 2.11 & 80 \\
\hline
\end{tabular}

New SuperKEKB collider is an upgraded KEKB which is placed in the same tunnel and utilizes many elements of the previous B-factory. The KEKB cells are kept in the High Energy Ring (HER). Dipole magnets in Low Energy Ring (LER) will be replaced, while other main magnets in the LER arcs are reused. The LER beam pipe will be completely replaced to a new one with ante-chambers.

Injector for KEKB is upgraded as well. Special damping ring is constructed to produce high intensive positron beams with low emittance.

At present most of the collider elements are produced and installation of them is going on. 


\section{The Belle II detector}

The Belle II detector should cope with the high rate of physical events and backgrounds and meet severe radiation background conditions. The detail description of the project is in the Technical Design Report. ${ }^{9}$ The schematic view of the Belle II detector (top half) in comparison to the previous Belle detector (bottom half) is presented in Fig. 1.

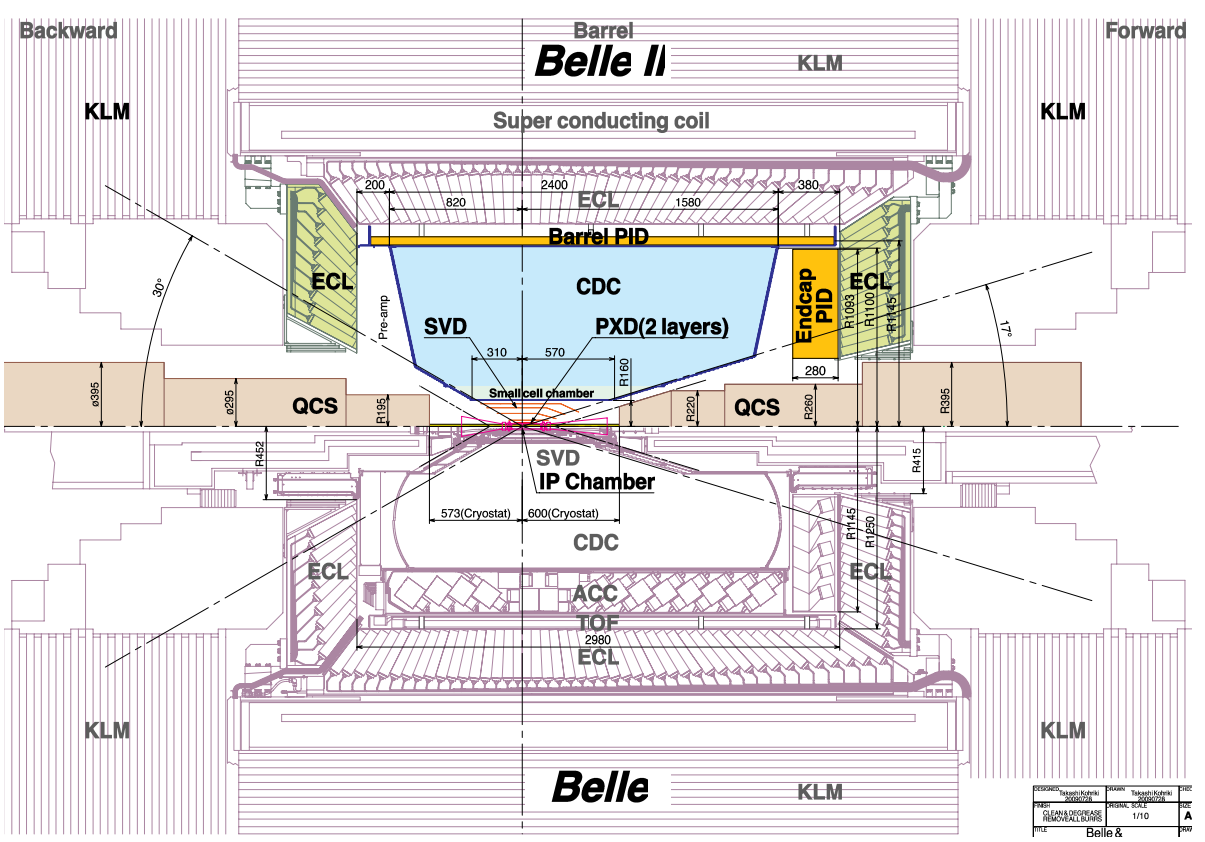

Fig. 1. Schematic view of the Belle II detector (top half) in comparison to the previous Belle detector (bottom half).

Vertex detector of the Belle II consists of the Pixel Detector (PXD) and Silicon Vertex Detector (SVD). The PXD is based on DEPFET technology which allows to produce very thin (down to $50 \mu \mathrm{m}$ ) sensors. Two layers of PXD with the pixel size of $50 \times 75 \mu \mathrm{m}$ will be arranged around the interaction region at the radial distances of 14 and $22 \mathrm{~mm}$ from the beam axis.

The SVD consists of four layers of double-sided silicon strip detectors (DSSD) arranged at the radial distances of 38, 80, 104 and $135 \mathrm{~mm}$. To increase radial coverage the slanted sensors are used in the forward region. The rectangular and wedge shape DSSDs with the strip pitch from $160 / 50 \mu \mathrm{m}$ to $240 / 75 \mu \mathrm{m}$ are used in SVD.

The PXD and SVD together should provide impact parameter resolution of about $20 \mu \mathrm{m}$. 


\section{B. A. Shwartz}

Main functions of the central drift chamber (CDC) are: track reconstruction and its precise momentum measurement; a measurement of the ionization losses of the charged particles for the identification purposes; a generation of the signals for the trigger system.

In comparison to Belle CDC, the radii of inner and outer cylinders changed from 77 to $160 \mathrm{~mm}$ and from 880 to $1130 \mathrm{~mm}$, respectively. The number of layers increases slightly, from 50 to 56 while the number of the sense wires changed from 8400 to 14336. The momentum resolution of the Belle II with CDC and SVD is:

$$
\sigma_{p_{t}} / p_{t}=0.11 \% \cdot p_{t}[\mathrm{GeV} / \mathrm{c}] \oplus 0.30 \% / \beta
$$

The expected $d E / d x$ resolution is about $5 \%$.

The particle identification system in the barrel part of the Belle II detector is based on the time-of-propagation (TOP) counters. ${ }^{10}$ The basic idea is to detect simultaneously the direction and arrival time of the Cherenkov photons propagating in the quartz radiator. Photons are detected by the Hamamatsu 16 channel MCPPMTs which have excellent timing and gain performance. This system will provide a good pion-kaon separation in the momentum range up to $3.5 \mathrm{GeV} / \mathrm{c}$.

Identification of charged particles in the forward endcap region will be performed by the proximity-focusing aerogel ring-imaging Cherenkov detector (ARICH). Cherenkov photons emitted in the $2 \mathrm{~cm}$ thick aerogel radiator with the refraction index $n \approx 1.05$ pass $20 \mathrm{~cm}$ expansion gap and are detected by the array of position sensitive hybrid avalanche photo-detectors (HAPD). This system allows to obtain $99 \%$ of kaon identification efficiency at $1 \%$ of pion misidentification for particles with $4 \mathrm{GeV} / \mathrm{c}$ momentum.

The baseline of the upgrade of the electromagnetic crystal calorimeter (ECL) is the electronics modification following the general strategy of the Belle upgrade. The main idea is to shorten the shaping time and to use a pipe-line readout with waveform analysis. However this may be not sufficient for proper performance of the endcap ECL parts where the background is extremely high. To improve this, a second stage of the upgrade is under developement. This modification implies a replacement of slow $\mathrm{CsI}(\mathrm{Tl})$ crystals to fast pure CsI. For the light readout vacuum photopenthodes will be used. The existing mechanical structure is kept. This option has been developing for the last five years and is now well prepared for construction.

A superconducting solenoid providing a magnetic field of $1.5 \mathrm{~T}$ as well as an iron yoke will be reused from the Belle detector.

The Belle KL\&Muon detector consisted of the glass-electrode resistive plate chambers (RPC). For the Belle II detector the end cap KL\&Muon parts as well as two innermost layers of the barrel KL\&Muon part are replaced to the system based on the plastic scintillators. Remaining barrel part keeps the RPC system. New KL\&Muon detectors consist of the polisterene scintillator bars with implemented WLS fibers for light collection. To detect the reemitted light delivered by fibers, MPPCs of $1.3 \times 1.3 \mathrm{~mm}$ area will be used. 
The data acquisition (DAQ) system of the Belle II detector has to cope with a high data flow which will reach $600 \mathrm{MB} / \mathrm{sec}$ at full luminosity. The detector front-end boards with digitizers are placed near or inside the detector structure and the digitized signals are transferred into COPPER systems through long optical fibers. A simple data reduction is performed on each front-end electronics board or on the receiver module called COPPER, while the data formatting and modulelevel event building is done on COPPER using the on-board CPU. The further event building and reduction are done on the readout PCs and the event builder, and finally the data are processed high level trigger system for the software event selection.

Comissioning of the SuperKEKB/Belle II is scheduled in three phases. During the phase 1, from the beginning of 2015, without Belle II and focussing quadrupoles, the machine tuning and vacuum scrubbibg as well as damping ring comissioning will be made. At the phase 2, from the middle of 2015, the Belle II detector will be installed without PXD. The physics run should start in 2016.

\section{Conclusion}

The last decade demonstrated the fruitfulness and efficiency of the flavor "factories" in particle physics. The huge amount of results was obtained at the Bfactories, but many new questions arose and the broad field of research will be opened by the superB-factory. It is clear that the superB-factory will produce the information complementary to the LHC. At present, the superKEKB/Belle II project is under construction and we can hope for new exciting results in the next decade.

\section{Acknowledgments}

I would like to thank organisers for the kind invitation to this impressive Conference as well as for their help and hospitality in Rome.

This work is supported in part by the Russian Education and Science Ministry, by FEDERAL TARGET PROGRAM "Scientific and scientific-pedagogical personnel of innovative Russia in 2009-2013", by agreement 14.B37.21.07777, by the Russian Fund for Basic Research grant 12-02-01296-a.

\section{References}

1. A. Abashian et al., Nucl. Instr. and Meth., A479, 117 (2002).

2. B. Aubert et al., Nucl. Instr. and Meth., A479, 1 (2002).

3. S. Kurokawa et al., Nucl. Instr. and Meth., A499, 1 (2003).

4. PEP-II Conceptual Design Report, SLAC-372, LBL-PUB-5303, CALT-68-1715, UCRL-ID-106426, UC-IIRPA-91-01 (1991).

5. Belle Collab. ( A. Abashian et al.), Phys. Rev. Lett. 86, 2509 (2001); BABAR Collaboration (B. Aubert et al. ), Phys. Rev. Lett. 86, 2515 (2001).

6. Belle Collab. ( A. Adachi et al.), Phys. Rev. Lett. 108, 171802 (2012). 
7. J. Beringer et al., Phys. Rev. D 86, 010001 (2012).

8. P. Raimondi, Talk, given at the $2 \mathrm{~d}$ SuperB workshop, Frascati, http://www.lnf.infn.it/ conference/superb06/talks/raimondi1.ppt, 2006.

9. T. Abe et al., Belle II technical design report, KEK Report 2010-1 (2010).

10. K. Inami et al., Nucl. Instr. and Meth. A595, 96 (2008). 